\title{
A HYBRID MODEL FOR TUMOR SPHEROID GROWTH IN VITRO I: THEORETICAL DEVELOPMENT AND EARLY RESULTS
}

\author{
YANGJIN KIM* and MAGDALENA A. STOLARSKA \\ School of Mathematics, University of Minnesota \\ Minneapolis, MN 55455, USA \\ HANS G. OTHMER \\ School of Mathematics and Digital Technology Center \\ University of Minnesota, Minneapolis, MN 55455, USA \\ othmer@math.umn.edu \\ Received 15 March 2007 \\ Revised 13 April 2007 \\ Communicated by N. Bellomo and P. K. Maini
}

\begin{abstract}
Tumor spheroids grown in vitro have been widely used as models of in vivo tumor growth because they display many of the characteristics of in vivo growth, including the effects of nutrient limitations and perhaps the effect of stress on growth. In either case there are numerous biochemical and biophysical processes involved whose interactions can only be understood via a detailed mathematical model. Previous models have focused on either a continuum description or a cell-based description, but both have limitations. In this paper we propose a new mathematical model of tumor spheroid growth that incorporates both continuum and cell-level descriptions, and thereby retains the advantages of each while circumventing some of their disadvantages. In this model the cell-based description is used in the region where the majority of growth and cell division occurs, at the periphery of a tumor, while a continuum description is used for the quiescent and necrotic zones of the tumor and for the extracellular matrix. Reaction-diffusion equations describe the transport and consumption of two important nutrients, oxygen and glucose, throughout the entire domain. The cell-based component of this hybrid model allows us to examine the effects of cell-cell adhesion and variable growth rates at the cellular level rather than at the continuum level. We show that the model can predict a number of cellular behaviors that have been observed experimentally.
\end{abstract}

Keywords: Tumor growth; hybrid model; mechanical effects; nutrient limitations.

AMS Subject Classification: 35Q80, 74L15, 92B05

*Present address: Mathematical Biosciences Institute, Ohio State University, Columbus, OH. †Present address: Department of Mathematics, University of St. Thomas, St. Paul, MN. 


\section{Introduction}

Avascular tumor growth in vivo is a complicated process that involves transport of nutrients from the surrounding tissue to the tumor, uptake of nutrients and release of waste products by the tumor, growth and division of individual cells, and cell-cell and cell-extracellular matrix (ECM) interactions via adhesion molecules. While it is possible to experimentally investigate individual biochemical and biomechanical processes involved in vitro, a complete understanding of tumor growth will only emerge when an integrated description of the in vivo processes can be formulated. This requires a mathematical model to weave the insights gained from experimental observations of individual steps into a coherent description of tumor growth. Existing mathematical models treat a tumor either as a continuum or as a discrete set of individual cells. Both of these approaches have their advantages and drawbacks. The former is easier to analyze both analytically and computationally, but suffers from the fact that it is difficult to incorporate details of cell-level behavior into the continuum description. In a cell-based model one can incorporate much more detail, but this leads to severe limitations on the number of cells that can be treated computationally. For instance, a tumor spheroid of $2 \mathrm{~mm}$ diameter contains $\sim 2 \times 10^{6}$ cells of $15 \mu \mathrm{m}$ diameter, and it is computationally prohibitive to treat these cells if each has a significant number of internal variables and interacts mechanically with its neighbors. Moreover, it is unnecessary to describe the quiescent and necrotic regions of a tumor with such detail, since it is primarily their mechanical and rheological properties that are important, and a similar statement applies to the medium surrounding a tumor. For these reasons we have developed a hybrid model that retains the cell-based description in the rapidly-proliferating regions at the tumor boundary, where it is advantageous to do so, and describes the remainder of the tumor and the ECM or surrounding gel as continua with possibly variable properties.

In this paper we focus on multicellular tumor spheroids (MCTSs), which are tumor cell aggregates grown in vitro that are used as a model of in vivo tumors because they closely mimic morphological and functional features of the latter. In an MCTS individual cells nucleate in a culture medium, and cells continue to proliferate until growth is arrested in the center of the spheroid, whereupon cells at the center may die and form a necrotic core (cf. Fig. 1(a)). Usually MCTS growth is avascular, and the necessary nutrients are supplied by diffusion from the surrounding tissue. Growth arrest and subsequent cell death typically occurs when the tumor diameter exceeds a critical value, and this is usually explained as resulting from diffusion limitations at the center, although other mechanisms may be involved. ${ }^{39}$ When there is a necrotic core, there is also usually another layer of quiescent cells, i.e. cells that are alive but not actively proliferating, around the necrotic core, and active proliferation is restricted to cells near the surface of the spheroid. In the hybrid model that is described later we model cells in the proliferating region of the MCTS as deformable ellipses, as was done earlier for the slug stage of the cellular slime mold Dictyostelium discoidium. ${ }^{42,18}$ The quiescent 

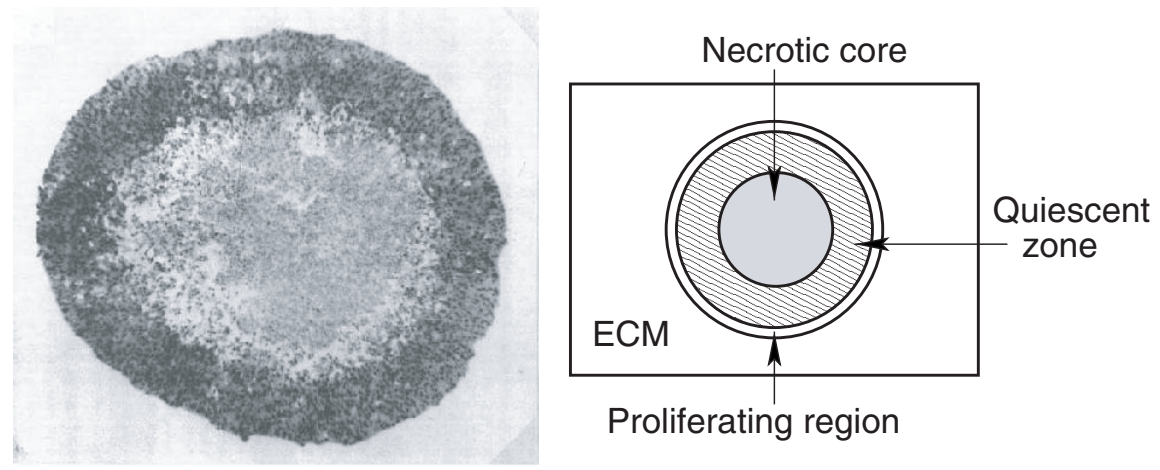

Fig. 1. (a) A typical multicellular tumor spheroid. The gray interior represents the necrotic core, the dark outer region the viable cells. From Sutherland et al. ${ }^{53}$ (b) A schematic of the model geometry.

and necrotic regions internal to the proliferating region and the extracellular matrix surrounding the spheroid are all modeled as viscoelastic continua (cf. Fig. 1(b)).

In the hybrid model only a few hundred actively-proliferating cells on the outer layer of spheroids are treated individually, and as a result, one can incorporate a great deal of detail about individual cells in the model. For instance, one can allow variations in the cell cycle time and in the effect of stress on growth of these cells, and one can incorporate variations in cell size, metabolic state and intraand intercellular mechanics. Since cells in the outer layers are discrete one can study the effect of changes in the balances between adhesion, chemotaxis and other effects on the rate of detachment of individual cells or small groups of cells from the tumor. This is important for predicting the spread of highly invasive tumors such as gliomas, for which the leading edge is diffuse and difficult to define precisely in a continuum description. ${ }^{27}$ In addition, the model can shed light on the question of whether there must be significant phenotypic differences between these invasive cells and other proliferating cells not at the leading edge, and whether cell-cycle-specific changes are involved. In one example, Landry ${ }^{33}$ showed (i) that rapidly-growing EMT6/Ro mammary tumor spheroids shed up to $1.5 \%$ of the total cells per hour, (ii) there is a positive correlation between the spheroid size and the rate of shedding, and (iii) cell shedding and the immediately following re-aggregation occur near the time of mitosis.

One of our objectives here is to understand how mechanics affects tumor growth. Recent experiments ${ }^{17,50}$ indicate that stress feedback from the mechanical processes can influence biochemical interactions and, therefore, growth in tissues. Two types of experiments of Helmlinger et al. ${ }^{29}$ further illustrate this effect. The first shows that when an MCTS is grown in an agarose gel the spheroid reaches an equilibrium size that depends on the stiffness of the gel. In the second type, an MCTS grown in an agarose-filled capillary tube adopts an ellipsoidal shape with the primary growth in the direction of the capillary axis because the stresses are anisotropic. 
These authors ruled out any effect of anisotropic diffusion of nutrients on growth and concluded that the spherical symmetry breaking is due to mechanical stresses. Here we use their experimental results to test the validity of our approach.

In the following section we briefly review existing models of avascular tumor growth, in Sec. 3 we introduce the new model and describe the computational algorithm, and in Sec. 4 we present some computational results.

\section{Previous Models of Growing Tumors}

The existing mathematical models of tumor growth are either pure cell-based models or pure continuum models. The former class can be further divided into singleor partial-cell lattice models or cell-based off-lattice models. In lattice models, the domain is subdivided into a lattice or grid of elements, and depending on the model, each "volume" element or node represents a single cell or a portion of a single cell. The interaction between these units is dictated by a fixed set of rules, typically prescribed in the form of a cellular automaton. Examples of this approach are given in Moreira and Deutsch ${ }^{38}$ and Drasdo et al. ${ }^{20}$ A particular example is the cellular Potts model, the defining characteristic of which is the set of rules that govern whether empty lattice cells will be occupied or not. These are based on a combination of cellular automaton rules and a local minimization of energy. ${ }^{54}$ Lattice-based models have been used to investigate various aspects of brain tumor growth, such as the shedding of cellular clusters from the primary tumor. ${ }^{35,7,34,60}$ This is a phenomenon that can be studied in more detail using the hybrid model developed later because the mechanical properties of the surrounding tissue can be incorporated. This is particularly important in the context of invasive brain tumors. ${ }^{33,28,52}$

In continuum models the cellular material in a tumor is described by a continuous density function, and continuum equations are used to model inter- and intracellular mechanical interactions and growth. Because the hybrid model described later can be viewed as a combination of an off-lattice model and a continuum model, we review previous mathematical applications of both types of models to tumor growth in more detail. Related descriptions are used in the context of models for angiogenesis, a process that often follows avascular growth, and these are reviewed in Mantzaris et $a l .{ }^{36}$

\subsection{Off-lattice single-cell models}

In off-lattice models each cell is treated as a unit of finite volume whose motion is not restricted to lattice points. In most models of this type the cell shapes have been restricted to spheres, ellipsoids, or Delaunay-decomposition-based shapes, with one exception mentioned later, in which arbitrary shapes in 2D are admitted. Mechanical interactions between the cells and between a cell and the surrounding matrix are governed by Newton's law, but the nature of the forces included varies widely between models. 
Off-lattice, cell-based models have been applied to a number of growing ${ }^{19,21}$ or actively-migrating ${ }^{42}$ tissues, and specific application to tumor growth have appeared. ${ }^{22,26,25}$ Drasdo and Höhme ${ }^{22}$ compare the growth of MCTSs and tumor monolayers using a model based on spherical cells for which the interactions between cells and between cells and substrate are modeled by interaction potentials, and the motion and growth of cells is simulated using a Metropolis algorithm. From the results of their numerical simulations they suggest that the shift from exponential to linear growth in monolayers with access to abundant nutrient can be attributed to cell-cell contact-mediated growth inhibition; cells in the interior of the monolayer are sufficiently compressed that their growth is inhibited.

Using a similar model, Galle et $a ._{.}{ }^{26}$ investigate the effects of (i) cell-cell contactmediated growth inhibition, (ii) cell-substrate contact-dependent cell cycle arrest, and (iii) cell-substrate-dependent programmed cell death (anoikis) on monolayer cell growth. They find that all three mechanisms must be "on" in order for the monolayer to persist, and conclude that the inactivation of any one of these mechanisms in an epithelial sheet may lead to uncontrolled growth and epithelial tumors. The results of both investigations ${ }^{22,26}$ shed light on tumor growth, but in both cases the cell population sizes are restricted to about $10^{5}$ cells, and the effect of the surrounding extracellular space is not considered.

The model of Schaller and Meyer-Hermann ${ }^{49}$ is similar to that of Galle et al. ${ }^{26}$ but employs a Voronoi tessellation based on a cell's center and radius to determine cellular deformation and contact area. Their model incorporates a detailed description of the cell cycle, and they assume that necrosis is due only to nutrient depletion and that quiescence is due only to cellular tension. The model reproduces the transition from exponential growth to polynomial growth that is seen in experiments, and for different nutrient concentrations it predicts the number of cells in each phase of the cell cycle.

In a novel 2D model Rejniak ${ }^{46}$ uses the immersed boundary method to model cells within a growing tumor. Cells are described by a set of discrete points connected by elastic springs, and the ensemble of points and springs is immersed in a viscous fluid that accounts for the material properties of the cells and the intercellular space. Cell-cell interactions are also represented by linear springs connecting boundary points on two distinct cells. This model describes the individual cell morphology very well, but because the immersed boundary method is computationally expensive, the model is restricted to around $10^{3}$ tumor cells in $2 \mathrm{D}$, and would be prohibitively expensive in $3 \mathrm{D}$.

\subsection{Continuum models}

When viewed as a continuum, a growing tumor can be thought of as a heterogeneous, multiphase, multicomponent mixture of growing, deformable material wherein the growth of one phase results from the uptake of mass from another. Mixture and multiphase theories have a long history in continuum mechanics, ${ }^{8,41,10}$ 
and in the last decade various models of tumor growth have been based on either a multiphase or a multicomponent description. ${ }^{43,44,32,55-57,11,12}$ Despite the fact that such models are biologically more realistic, there are serious technical difficulties in experimentally measuring quantities such as the partial displacements, relative velocities and the partial stresses necessary to develop suitable constitutive relations for multicomponent and/or multiphase systems. The problem is somewhat easier in multiphase models if one adopts a Darcy-like law for the cell-fluid interactions, but this simply postulates away some of the difficulties without solving them. Singlephase models, while not as realistic in many respects, capture many important characteristics of tumor growth without the complications of a multi-component or multiphase model. Here we briefly describe some single-phase models that involve mechanical aspects of tumor growth and multiphase models that address the experiments of Helmlinger et al. ${ }^{29}$; a more extensive review can be found in Araujo and McElwain. ${ }^{3}$

A number of models are based on the work of Jones et al., ${ }^{31}$ wherein the tumor is comprised entirely of proliferating cells. These authors assumed that both growth and the material response are isotropic and that the material response is hypoelastic, i.e. the strain rate is directly proportional to the stress rate. The model predicts that the tumor reaches a nutrient-controlled steady-state size, but the stress does not, which is physically unrealistic. This problem arises from the fact that the stress depends on the velocity of tumor evolution, and even when the velocity is zero at the tumor boundary, thereby leading to a tumor of equilibrium size, the velocities are not necessarily zero in the interior of the tumor.

Araujo and McElwain ${ }^{4}$ modified this model by introducing anisotropic growth, albeit in a spherically symmetric framework. In this model, if the circumferential stress $\sigma_{\theta}$ is less than the radial stress $\sigma_{r}$, then growth in the radical direction, dominates, while if $\sigma_{r}<\sigma_{\theta}$, growth in the circumferential direction increases. This criterion is introduced into the constitutive equation and mass balance equation through the introduction of strain-multiplier functions. These modifications of the constitutive equation and the growth assumptions lead to the prediction that the stress reaches a steady state when the tumor reaches an equilibrium size. This modified model was applied to a vascularized tumor growing nonhomogeneously in free suspension in order to explain blood vessel collapse due to residual stress. ${ }^{5}$ They find that once the vessel collapse front reaches the tumor boundary, the tumor regresses. In more recent work ${ }^{6}$ the authors consider the effect of residual stresses on vessel collapse for a nonhomogeneously growing spherical tumor embedded in an extracellular matrix. In this case, the tumor does not regress, but rather reaches a quasi-steady size. The comparison of these two models illustrates the effect that residual stresses have on growth, and indicates the importance of understanding mechanical effects on tumor growth.

Ambrosi and Mollica ${ }^{1}$ incorporate growth by a multiplicative decomposition of the deformation gradient tensor into elastic and growth factors, a decomposition used earlier by Hoger ${ }^{30}$ in the context of growing tissues. The material properties of the tumor are described by an elastic Blatz-Ko strain energy function, and 
this description is later used $^{2}$ to simulate the radially symmetric experiment of Helmlinger et al. ${ }^{29}$ While the simulations capture the general qualitative behavior of tumor growth in agarose gel of increasing stiffness, the model does not predict the experimentally observed difference in equilibrium sizes for different agarose concentrations.

Chen et al. ${ }^{16}$ extend earlier work of Landman and Please ${ }^{32}$ in the form of a onedimensional version of the spherically symmetric experiment of Helmlinger et al. Their model correctly reproduces the experimental observation ${ }^{29}$ that the steadystate tumor size decreases in a stiffer medium. Once the tumor is removed from the elastic medium and is allowed to grow in a free suspension, linear growth resumes, as is observed experimentally. A novel aspect of the model by Landman and Please, and therefore also of that of Chen et al., is that necrosis is assumed to be caused by mechanical stresses. The model also predicts that the stress from the surrounding medium delays the onset of necrosis, but neither of these predictions has been observed experimentally.

In Roose et $a .^{47}$ a poroelastic model is used to explain the effect of stress on a growing tumor that is embedded in an agarose matrix. Mass exchange from the fluid phase to the cellular phase depends on the oxygen concentration in the fluid phase and the stress in the cell phase. Specifically, the stress dependence is included via the function $1-\beta \overline{\boldsymbol{\sigma}}$, where $\overline{\boldsymbol{\sigma}}$ is the average of the bulk stresses. In a one-dimensional, spherically symmetric reduction of their full model, Roose et al. show that the equilibrium size of the spherically symmetric tumor decreases as the stiffness of the matrix increases. In addition, their experiments show that the change in equilibrium size of the spheroid is partially due to a decrease in size of individual cells as the stiffness of the matrix increases.

While the models described above have been used to investigate various important aspects regarding tumor growth, there still are many issues to address. Most previous models deal with tumor growth in the absence of a mechanically-resistant extracellular matrix. Those that incorporate the extracellular matrix into the model are continuum models and cannot address how the material properties of the extracellular matrix affects the active motion of cells and the morphology of the tumor. The novel combination of continuum and discrete approaches incorporated in the hybrid model allows one to investigate this effect and others.

\section{The Hybrid Model}

There are up to four geometrically-distinct regions in the hybrid model, the extracellular matrix or agarose gel surrounding the tumor, a shell of actively-proliferating cells at the outer edge of the tumor, a quiescent zone bordering the activelyproliferating region, and a necrotic core (cf. Fig. 2). We denote these $\mathcal{G}, \mathcal{P}, \mathcal{Q}$, and $\mathcal{N}$, respectively. The latter two are only present in a sufficiently large tumor, but for generality we describe the situation in which all four regions are present. The actively proliferating region comprises a layer $3-5$ cells thick in the radial direction, and therefore contains a few hundred cells. We assume that the quiescent layer has 


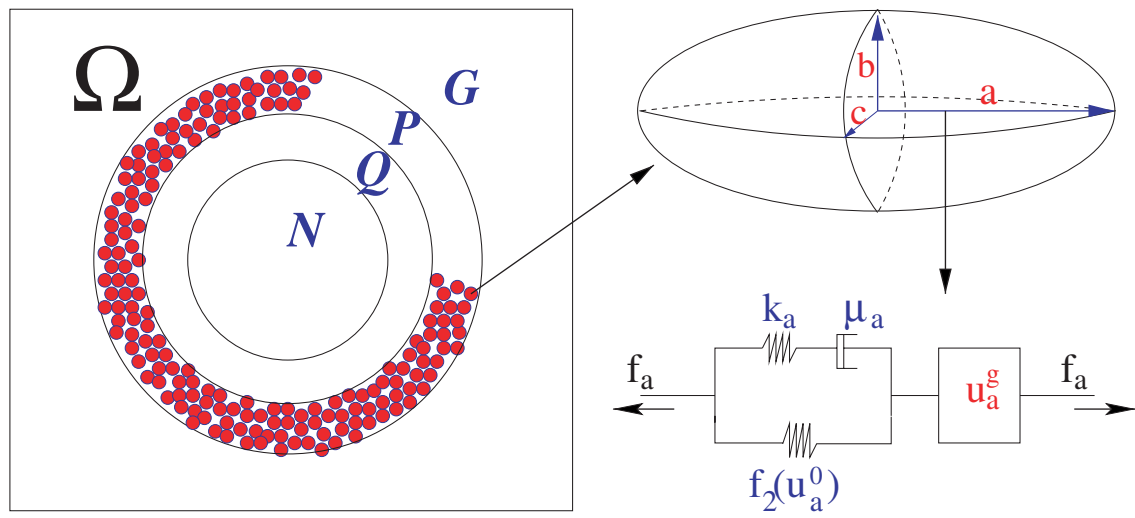

Fig. 2. A schematic showing the notation used for the subdomains, the representation of cells in the proliferating zone as ellipsoids, and the representation of the Kelvin and growth elements that characterize the internal rheology of each cell in $\mathcal{P}$.

constant thickness, as has been observed experimentally. ${ }^{24}$ Therefore, the computational domain $\Omega$ consists of the union of the four disjoint sub-domains $\mathcal{G}, \mathcal{P}, \mathcal{Q}, \mathcal{N}$, the latter two of which may be empty. We assume that the outer gel, the quiescent region, and the necrotic region are homogeneous materials as others have proposed (cf. Refs. 9, 48, 45 and 59 and therein for more details), but different material parameters are used in $\mathcal{G}, \mathcal{Q}$ and $\mathcal{N}$. There is no computational difficulty in treating different material properties in adjacent regions such as $\mathcal{Q}$ and $\mathcal{N}$; one simply has to match forces and displacements across the moving boundary. However, the boundaries between the cell-based region $\mathcal{P}$ and the continuum regions $\mathcal{G}$ and $\mathcal{Q}$ are very irregular, and rather than attempting to describe them in full detail in this model we create two artificial boundaries across which the forces are transmitted, as shown in Fig. 3. This will be described in detail later.

The reaction-diffusion equations for oxygen and glucose, the primary nutrients considered here, are solved on the entire domain $\Omega$. It is also assumed that the concentrations of nutrients are fixed at the outer boundary, and thus Dirichlet boundary conditions are imposed there. The necrotic region $\mathcal{N}$ is defined as a subdomain of the tumor interior in which the appropriate nutrient levels are below specified thresholds. This is a simplification of the real situation, in which the geometry of the interface between $\mathcal{Q}$ and $\mathcal{N}$ is not precisely defined. Different diffusion coefficients are used on each region and the uptake function is taken into consideration only in tumor regions.

The proliferating zone $\mathcal{P}$ comprises a few hundred cells that grow and divide as dictated by nutrient conditions, and whose shape changes are governed by their internal rheology and the forces acting on them. We assume that cells grow as long as they are within the threshold of stress and have adequate nutrients. Some of the cells in $\mathcal{P}$ become quiescent when the level of nutrients drops below the threshold, and since the quiescent region $\mathcal{Q}$ is represented as a continuum, this requires that 

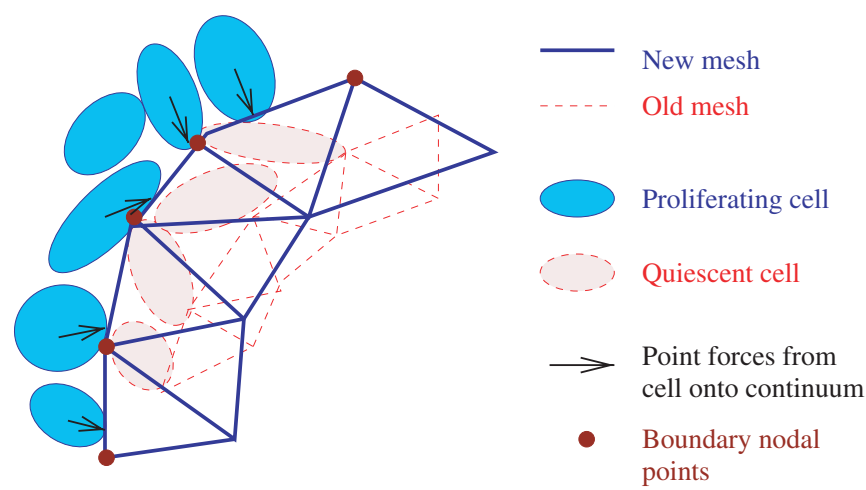

Fig. 3. A schematic showing how forces are transmitted from individual cells in the proliferating region to the quiescent region, which is treated as a continuum. A similar schematic applies to the interface between the proliferating region and the surrounding gel. When we transform the part of proliferating region to the quiescent region, we interpolate the data from the old mesh to the new mesh using information stored at nodal points of the old mesh and at the center of the transformed cells (i.e. quiescent cells) in the cell-based model. This data transfer is done using a least squares projection.

those cells be transformed into the continuum region $\mathcal{Q}$. The displacements of these transformed cells and the forces acting on them are converted into displacements and stress fields in this newly formed continuum material in $\mathcal{Q}$. We also assume that the ECM between cells that are converted into continuum is converted as well so as to preserve mass during the transformation.

\subsection{The cell-based component}

There are three major constituents that are needed to describe individual cells: (i) their mechanical interaction with the surroundings, (ii) how an individual cell reacts to forces on it, and (iii) how growth and division are described, and how stress affects growth. These are described in the following two subsections.

\subsubsection{The forces acting on individual cells}

The mechanical behavior of individual cells in the proliferating zone is based on the model developed by Dallon and Othmer ${ }^{18}$ (hereafter the paper and model is denoted DO), which is the extension to $3 \mathrm{D}$ of an earlier model. ${ }^{42}$ The new aspect that is needed in the present context is the growth and division of cells.

The forces on a cell in the DO model are (i) the active forces exerted on neighboring cells or the substrate, (ii) a reactive force due to forces exerted by other cells on it, (iii) the dynamic drag forces that arise as a moving cell forms and breaks adhesive bonds with neighboring cells, and (iv) a static frictional force that exists when cells are rigidly attached to each other or to the substrate. The active force on cell $i$ is denoted $\mathbf{T}_{i, j}$, wherein $j=0$ denotes the substrate, and the reaction force to this is denoted $\mathbf{M}_{j, i}$. The static force, which is denoted $\mathbf{S}_{j i}$, is the binding 
force on the $i$ th cell when bound to the $j$ th. Since $\mathbf{S}_{j i}=-\mathbf{S}_{i j}$, the cell-cell forces cancel on all but those cells attached to the substrate (a more detailed discussion of all forces involved is given in DO).

The total force on the $i$ th cell is then given by

$$
\mathbf{F}_{i}=\sum_{j \in \mathcal{N}_{i}^{a}} \mathbf{M}_{j, i}+\sum_{j \in \mathcal{N}_{i}^{a}} \mathbf{T}_{j, i}+\sum_{j \in \mathcal{N}_{i}^{d}} \mu_{i j}\left(\mathbf{v}_{j}-\mathbf{v}_{i}\right)+\sum_{j \in \mathcal{N}_{i}^{s}} \mathbf{S}_{j i},
$$

where $\mathcal{N}_{i}^{a}$ denotes the neighbors of $i$, including the substrate, upon which it can exert traction, $\mathcal{N}_{i}^{d}$ is the set of "cells" (which includes substrate and extracellular matrix) that interact with $i$ via a frictional force, and $\mathcal{N}_{i}^{s}$ denotes the set of cells that statically bind to cell $i$.

\subsubsection{The rheology of the cytoplasm and the effect of stress on growth}

The intracellular reaction to these forces is treated as in the DO model, i.e. the cells are treated as oriented ellipsoids whose cytoplasm is described as an incompressible viscoelastic solid. In the present context we must add growth and division to the passive response. In this paper we do not include chemotaxis driven by active motive forces, and these terms are henceforth omitted from (3.1). Certainly some cells may detach from the tumor and migrate through the surrounding tissue, and this will be investigated in the future.

As in the DO model, when cells do not grow their volume is constant under all deformations, but when there is growth this constraint does not apply. We define $V_{0}$ as the volume cells attain immediately after division, and we assume here that this is the same for all cells. It will be clear later that this is easily relaxed. We also suppose that stress and nutrient levels affect the growth rate, and that the effect of stress is isotropic. The latter implies that in the absence of external forces all cells relax to a spherical shape whatever their initial shape, whether or not there is growth. In the absence of nutrient or stress limitations cells grow to the volume $2 V_{0}$ and then instantly divide into equal two daughter cells. The minimal cell-cycle time $\tau_{c}$ is the doubling time under these conditions. In the presence of extracellular forces the orientation of cell division is determined by the direction of the net force exerted on the cell, as others have assumed. ${ }^{26}$

In the context of a tumor or other tissue, cells interact with neighboring cells and deplete the nutrients, and this may lead to nonuniform growth in the population and an increased cell-cycle time. Stress and nutrient levels can affect the growth of cells differently because the former effect need not be isotropic while the latter always is. Without adequate nutrients (here oxygen and glucose) cells enter the quiescent phase. If the nutrient level drops too low they die or undergo apoptosis, and in a tumor this leads to the necrotic core. Of course apoptosis may occur throughout the proliferating and quiescent zones as well, but this is not included here.

We assume that growth stops if the stress is too large in magnitude, as has been suggested by others, and this effect is directional in nature. Without the growth 
component, the governing equations for the length of an axis of a cell reduce to those in the DO model. In this case the response of the Kelvin element to a step change in force is creep to the viscous limit after the initial elastic jump, followed by return to the resting length after removal of the external force. If the applied force is too compressive or tensile in the current model, the response to the applied force is the same as in the DO model, but if the magnitude is small enough the displacement of the solid-growth element increases and asymptotes to the linear growth profile that results from the growth component (Fig. 4(b)). Release of the external force leads to the resting length of the spring-dashpot component but the nontrivial growth component leads to an increase in the length.

The governing equations of the length of the $i$ th axis, $i=\mathrm{a}, \mathrm{b}, \mathrm{c}$, of a cell are

$$
\begin{aligned}
u_{i} & =u_{i}^{0}+u_{i}^{g}, \\
\left(u_{i}^{0}\right)^{\prime} & =\left(\frac{k_{i}}{\mu_{i}}\left[f_{i}(t)+\bar{p}-f_{2}\left(u_{i}^{0}\right)\right]+f_{i}^{\prime}(t)\right) \times\left(\frac{d f_{2}\left(u_{i}^{0}\right)}{d u_{i}^{0}}+k_{i}\right)^{-1}, \\
\left(u_{i}^{g}\right)^{\prime} & =f\left(f_{i}(t)+\bar{p}\right),
\end{aligned}
$$

where $u_{i}$ is the change in the length of the $i$ th axis, $u_{i}^{0}\left(u_{i}^{g}\right)$ is the change in the length of the $i$ th axis due to a change in the passive (growth) element, $f_{2}$ is the nonlinear spring force from the spring in parallel, $f_{i}$ is the magnitude of the force applied at each end, $\mu_{i}$ is the viscous coefficient of the dash-pot, $k_{i}$ is the spring constant for the spring in the Maxwell element, $\bar{p}$ is the force due to pressure, and $f$ is the growth function (cf. Fig. 4(a)). The specific form of the function $f_{2}$ and details of how these equations are established are given in DO.

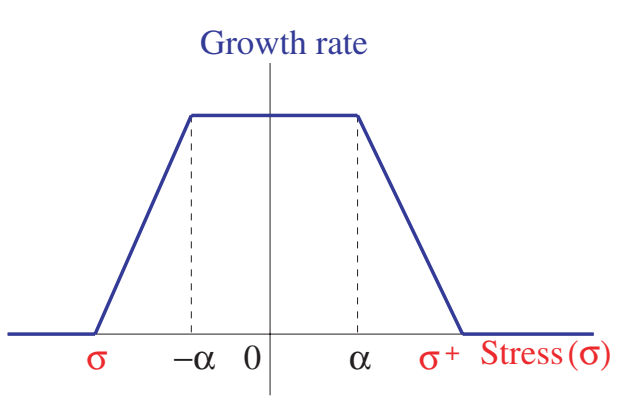

(a)

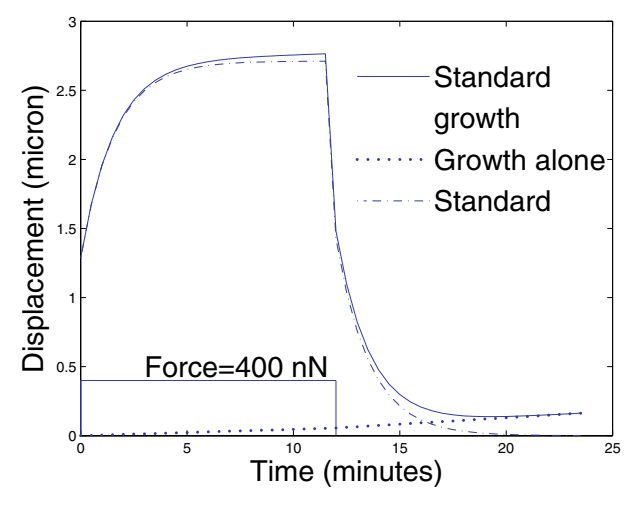

(b)

Fig. 4. (a) The growth rate function $f(\sigma)$. (b) The creep function for the rheological model in Fig. 2, using the parameters given in Table 1. A force of magnitude $400 \mathrm{nN}$ is applied at time zero and removed at $12 \mathrm{~min}$. The dashed-dotted line shows the response of the Kelvin element, the dotted line shows the response due to growth, and the solid line shows the combined effect. 
It is assumed that the passive response is incompressible, and therefore the three equations for $u_{a}^{0}, u_{b}^{0}$, and $u_{c}^{0}$ are solved with the volume constraint

$$
\left(u_{a}^{0}\right)^{\prime}\left(u_{b}^{0}+b_{0}^{*}\right)\left(u_{c}^{0}+c_{0}^{*}\right)+\left(u_{a}^{0}+a_{0}^{*}\right)\left(u_{b}^{0}\right)^{\prime}\left(u_{c}^{0}+c_{0}^{*}\right)+\left(u_{a}^{0}+a_{0}^{*}\right)\left(u_{b}^{0}+b_{0}^{*}\right)\left(u_{c}^{0}\right)^{\prime}=0
$$

where $a_{0}^{*}, b_{0}^{*}$ and $c_{0}^{*}$ are the lengths of three axes $\mathbf{a}, \mathbf{b}, \mathbf{c}$ after growth $\left(a_{0}^{*}=a_{0}+u_{a}^{g}\right.$, $b_{0}^{*}=b_{0}+u_{b}^{g}, c_{0}^{*}=c_{0}+u_{c}^{g}$ where $a_{0}, b_{0}$ and $c_{0}$ are the initial lengths of three axes). This means that the viscoelastic components on the three axes must satisfy the volume constraint after growth.

The effect of stress on growth is described by the relation $\dot{u}_{i}^{g}=f\left(\sigma_{i}\right)$, for $i=a, b, c$, where $\sigma_{i}$ is the axial component of the force applied along the $i$ th axis. The form of $f$ is based on previous experimental observations. For example, Roose et $a l .{ }^{47}$ showed that increased gel concentration induced decreased cell volume and postulated the relation

$$
\dot{u}_{a}^{g}=1-\beta \bar{\sigma} .
$$

Here we use a piecewise linear function and include the effect of tensile as well as compressive stresses (cf. Fig. 4). Thus we define $f(\cdot)$ as follows:

$$
f(\sigma)= \begin{cases}c^{-}\left(\sigma-\sigma^{-}\right) & \text {if } \sigma^{-} \leq \sigma \leq-\alpha, \\ -c^{+}\left(\alpha-\sigma^{+}\right) & \text {if }-\alpha \leq \sigma \leq \alpha, \\ -c^{+}\left(\sigma-\sigma^{+}\right) & \text {if } \alpha \leq \sigma \leq \sigma^{+}, \\ 0 & \text { if } \sigma>\sigma^{+}, \sigma<\sigma^{-},\end{cases}
$$

where $c^{+}, c^{-}$are positive constants, $\sigma^{+}>0, \sigma^{-}<0,\left[\sigma^{-}, \sigma^{+}\right]$is the interval of positive growth, $c^{+}\left(\alpha-\sigma^{+}\right)=-c^{-}\left(-\alpha-\sigma^{-}\right)$. In the simulations discussed later we use $\alpha=0$.

\subsubsection{The equations of motion}

As stated earlier, in this paper we do not consider an active force $\mathbf{T}_{i, j}$ and the reactive force $\mathbf{M}_{j, i}$. We further restrict attention to a two-dimensional system, and therefore Newton's law for the $i$ th cell reduces to

$$
\begin{aligned}
A_{i f} \mu_{f} \mathbf{v}_{i} & +A_{i s} \mu_{s} \mathbf{v}_{i}+\mu_{\text {cell }} \sum_{j \neq i} A_{i j}\left(\mathbf{v}_{i}-\mathbf{v}_{j}\right) \\
& +\frac{A}{6 \pi r_{i b}}\left(\mathbf{R}_{0, i}^{*}+\sum_{j \neq i} \mathbf{A}_{i, j}+\sum_{j \neq i} \mathbf{R}_{j, i}+\sum_{j \neq i} \mathbf{R}_{j, i}^{*}\right)=0 .
\end{aligned}
$$

Here $A=A(t)$ is the total area of an undeformed cell, $A_{i j}=A_{i j}(t), A_{i f}=A_{i f}(t)$, $A_{i s}=A_{i s}(t)$ are the lengths of contact regions between cell $i$ and cell $j$, cell $i$ and the interstitial fluid or matrix, and cell $i$ and the substrate at time $t$ respectively, $\mu_{\text {cell }}\left(\right.$ resp. $, \mu_{s}, \mu_{f}$ ) is the degree of adhesiveness between the cells (resp., between the substrate and the cells, and the fluid viscosity), $r_{i b}=u_{b}+b_{0}$, and $\mathbf{v}_{i}$ is the velocity 
Table 1. Parameters for the cell-based component of the model.

\begin{tabular}{|c|c|c|c|}
\hline Parameter & Description & Value & Refs. \\
\hline \multicolumn{4}{|c|}{ Adhesion parameters } \\
\hline$\mu_{\text {cell }}$ & Cell-cell adhesiveness & 27.0 dyn $\mathrm{s} / \mathrm{cm}$ & 18 \\
\hline$\mu_{s}$ & Cell-substrate adhesiveness & 27.0 dyn s/cm & 18 \\
\hline$\mu_{f}$ & The fluid viscosity & 2.7 dyn s/cm & 18 \\
\hline \multicolumn{4}{|c|}{ Rheological parameters } \\
\hline$c^{+}$ & Growth function parameter & $5.16089 \times 10^{-9} \mathrm{~mm} /(\min . \mathrm{nN})$ & \\
\hline$\sigma^{+}$ & Growth function parameter & $800 \mathrm{nN}$ & \\
\hline$\sigma^{-}$ & Growth function parameter & $-4 \mathrm{nN}$ & \\
\hline$\alpha$ & Growth function parameter & $0.0 \mathrm{nN}$ & \\
\hline$k_{a}$ & Standard solid parameter & $163.8 \mathrm{dyn} / \mathrm{cm}$ & 18 \\
\hline$k_{2}$ & Standard solid parameter & $147.5 \mathrm{dyn} / \mathrm{cm}$ & 18 \\
\hline$\mu_{a}$ & Standard solid parameter & 123 dyn $\min / \mathrm{cm}$ & 18 \\
\hline
\end{tabular}

of cell $i$. The base parameters that characterize the cells are given in Table 1; those that are changed later will be noted. Details as to how the various terms in (3.7) are computed can be found in DO. Of course here the total number of cells changes due to cell division and the incorporation of cells into the quiescent region.

\subsection{The continuum components for mechanics and nutrients}

As was indicated in the Introduction, we use a continuum description for the mechanical response of the gel outside the tumor, and for the quiescent and necrotic regions of the tumor. The outer gel $\Omega_{0}$ and the interior regions $\Omega_{m}, m=$ 1,2 , are treated as linear viscoelastic materials with different material properties $\mathcal{C}^{m}, \mathcal{D}^{m}, m=0,1,2$. Therefore the constitutive equations are

$$
\boldsymbol{\sigma}=\mathcal{C} \boldsymbol{\epsilon}+\mathcal{D} \dot{\boldsymbol{\epsilon}} \quad \text { on } \Omega \times(0, T),
$$

where the strain is defined as

$$
\boldsymbol{\epsilon}=\frac{1}{2}\left(\nabla \mathbf{u}+(\nabla \mathbf{u})^{\mathrm{T}}\right)
$$

$\mathbf{u}$ is the displacement field, and "." is the partial derivative with respect to time. $\mathcal{C}$ and $\mathcal{D}$ are defined implicitly by the relations

$$
\begin{array}{rlrl}
(\mathcal{C} \boldsymbol{\epsilon})_{\alpha \beta}=\frac{E}{1+\nu} \boldsymbol{\epsilon}_{\alpha \beta}+\frac{E \nu}{1-\nu^{2}}\left(\boldsymbol{\epsilon}_{11}+\boldsymbol{\epsilon}_{22}\right) \delta_{\alpha \beta}, & & 1 \leq \alpha, \beta \leq 2, \\
(\mathcal{D} \dot{\boldsymbol{\epsilon}})_{\alpha \beta}=\mu_{1} \dot{\boldsymbol{\epsilon}}_{\alpha \beta}+\mu_{2}\left(\dot{\boldsymbol{\epsilon}}_{11}+\dot{\boldsymbol{\epsilon}}_{22}\right) \delta_{\alpha \beta}, & 1 \leq \alpha, \beta \leq 2 .
\end{array}
$$

Here $E$ and $\nu$ are Young's modulus and the Poisson ratio, respectively, and $\mu_{1}, \mu_{2}$ are the shear and bulk viscosities, respectively. These are different in each of the regions (cf. Table 2), but for simplicity we omit the additional label needed on the parameters. 
We neglect inertial effects and therefore the momentum equation reduces to

$$
\nabla \cdot \boldsymbol{\sigma}=0 \quad \text { on } \Omega \times(0, T)
$$

with boundary conditions

$$
\begin{array}{rlll}
\mathbf{u}^{0}=0 & \text { on } & \Gamma_{0} \times(0, T), \\
\boldsymbol{\sigma}^{0} \cdot \mathbf{n}=\mathbf{q}_{0} & \text { on } & \Gamma_{c 0} \times(0, T), \\
\boldsymbol{\sigma}^{1} \cdot \mathbf{n}=\mathbf{q}_{1} & \text { on } & \Gamma_{c 1} \times(0, T) .
\end{array}
$$

Here $\Gamma_{0}$ is the fixed outer boundary, $\Gamma_{c 0}$ is the interface between $\mathcal{G}$ and $\mathcal{P}, \Gamma_{c 1}$ is the interface between $\mathcal{P}$ and $\mathcal{Q}, \mathbf{u}^{0}$ and $\mathbf{u}^{1}$ are the displacement fields on $\mathcal{G}$ and $\mathcal{P}$, resp., $\boldsymbol{\sigma}^{0}$ and $\boldsymbol{\sigma}^{1}$ are the stress fields on $\Omega_{0}$ and $\Omega_{1}$, resp. $\mathbf{q}_{0}$ and $\mathbf{q}_{1}$ are boundary forces acting on $\Gamma_{c 0}$ and $\Gamma_{c 1}$ resp. ; these are calculated from the cellbased component as indicated in Fig. 3. These equations are solved using the finite element method based on the triangular mesh shown in Fig. 5(a). The parameters used in the computations are given in Table 2.

The nutrients considered here are oxygen and glucose, and we assume that their consumption is described by Michaelis-Menten kinetics. The governing equations for the evolution of the nutrients, assuming Dirichlet boundary conditions, are

$$
\begin{aligned}
\frac{\partial c_{\mathrm{O}_{2}}}{\partial t}=D_{\mathrm{o}} \nabla^{2} c_{\mathrm{O}_{2}}-\phi_{\mathrm{O}_{2}}\left(c_{\mathrm{O}_{2}}\right)\left(A_{\mathrm{O}_{2}}+\frac{B_{\mathrm{O}_{2}}}{c_{\mathrm{gl}}+n_{\mathrm{O}_{2}}}\right)\left(\frac{c_{\mathrm{O}_{2}}}{c_{\mathrm{O}_{2}}+k_{\mathrm{O}_{2}}}\right) & & \text { in } \Omega \\
\frac{\partial c_{\mathrm{gl}}}{\partial t}=D_{\mathrm{g}} \nabla^{2} c_{\mathrm{gl}}-\phi_{\mathrm{gl}}\left(c_{\mathrm{gl}}\right)\left(A_{\mathrm{gl}}+\frac{B_{\mathrm{gl}}}{c_{\mathrm{O}_{2}}+n_{\mathrm{gl}}}\right)\left(\frac{c_{\mathrm{gl}}}{c_{\mathrm{gl}}+k_{\mathrm{gl}}}\right) & & \text { in } \Omega \\
c_{\mathrm{O}_{2}}=c_{\mathrm{O}_{2}}, \quad c_{\mathrm{gl}}=c_{\mathrm{gl}}^{-} & & \text {on } \partial \Omega
\end{aligned}
$$

where $c_{\mathrm{O}_{2}}\left(c_{\mathrm{gl}}\right)$ is the molar concentration of oxygen (glucose), the second term of each equation is a function describing the consumption of oxygen (glucose) by the

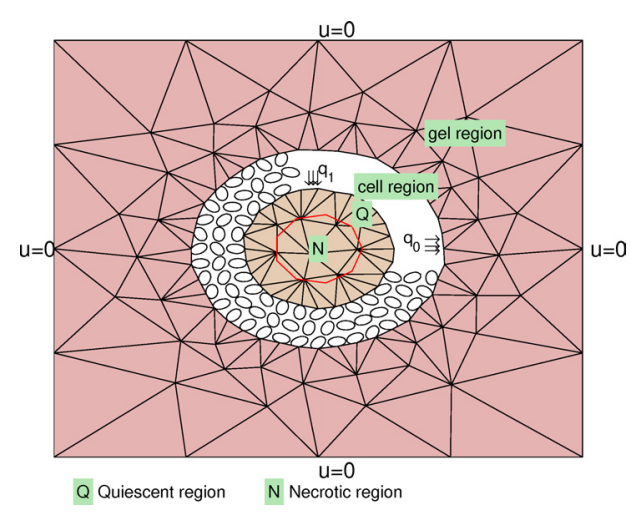

(a)

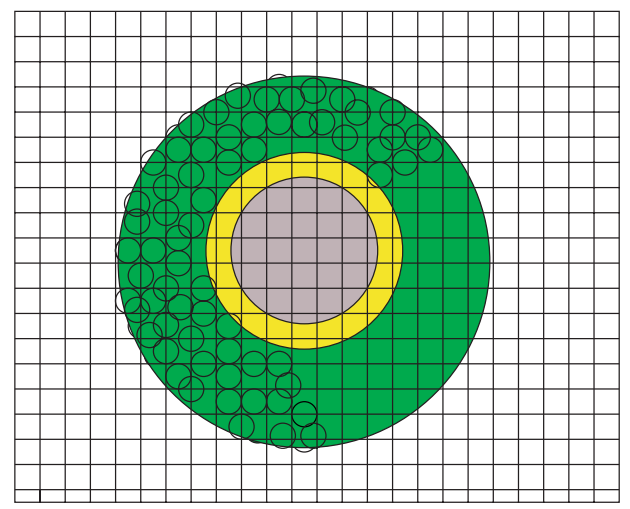

(b)

Fig. 5. The computational grid for solving the equations of motion on the continuum components of the tumor (a) and for the reaction-diffusion equations (b). 
Table 2. The rheological parameters for the continuum regions.

\begin{tabular}{clll}
\hline Parameter & \multicolumn{1}{c}{ Description } & \multicolumn{1}{c}{ Value } \\
\hline $\mathcal{G}$ & & Refs. \\
& $E^{a}$ & Young's modulus $E$ in $\mathcal{G}$ & $10-200 \mathrm{MPa}$ \\
& $\nu^{a}$ & Poisson ratio in $\mathcal{G}$ & 0.5 \\
$\mu_{1}^{a}$ & Shear viscosity in $\mathcal{G}$ & $100.0(\mathrm{nN} \cdot \mathrm{min}) /(\mathrm{mm})^{2}$ & \\
$\mu_{2}^{a}$ & Bulk viscosity in $\mathcal{G}$ & $300.0(\mathrm{nN} \cdot \mathrm{min}) /(\mathrm{mm})^{2}$ \\
$\mathcal{Q}$ & & \\
& & \\
$E^{q}$ & Young's modulus $E$ in $\mathcal{Q}$ & $100 \mathrm{MPa}$ \\
$\nu^{q}$ & Poisson ratio in $\mathcal{Q}$ & 0.5 \\
$\mu_{1}^{q}$ & Shear viscosity in $\mathcal{Q}$ & $100.0(\mathrm{nN} \cdot \mathrm{min}) /(\mathrm{mm})^{2}$ \\
$\mu_{2}^{q}$ & Bulk viscosity in $\mathcal{Q}$ & $300.0(\mathrm{nN} \cdot \mathrm{min}) /(\mathrm{mm})^{2}$ \\
$\mathcal{N}$ & & \\
$E^{n}$ & Young's modulus $E$ in $\mathcal{N}$ & $80 \mathrm{MPa}$ \\
$\nu^{n}$ & Poisson ratio in $\mathcal{N}$ & 0.5 \\
$\mu_{1}^{n}$ & Shear viscosity in $\mathcal{N}$ & $80.0(\mathrm{nN} \cdot \min ) /(\mathrm{mm})^{2}$ \\
$\mu_{2}^{n}$ & Bulk viscosity in $\mathcal{N}$ & $250.0(\mathrm{nN} \cdot \mathrm{min}) /(\mathrm{mm})^{2}$ \\
\hline
\end{tabular}

tumor, $D_{\mathrm{o}}\left(D_{\mathrm{g}}\right)$ is the space-dependent $(\mathcal{G}, \mathcal{P}, \mathcal{Q}, \mathcal{N})$ diffusion coefficient of oxygen (glucose), $A_{\mathrm{O}_{2}}, A_{\mathrm{gl}}, B_{\mathrm{O}_{2}}, B_{\mathrm{gl}}, k_{\mathrm{O}_{2}}, k_{\mathrm{g}}, n_{\mathrm{O}_{2}}$, and $n_{\mathrm{gl}}$ are empirically determined parameters, and the cell consumption indicator function is given by

$$
\phi_{\mathrm{O}_{2}}\left(c_{\mathrm{O}_{2}}\right)= \begin{cases}1 & \text { in } \mathcal{P}, \mathcal{Q} \\ 0 & \text { in } \mathcal{G}, \mathcal{N}\end{cases}
$$

and similarly for $\phi_{\mathrm{gl}}\left(c_{\mathrm{gl}}\right)$. The parameter values for the reaction-diffusion equations are given in Table 3.

The reaction-diffusion equations (3.11) are solved on the regular grid using an alternating-direction implicit (ADI) scheme and the nonlinear solver nksol for algebraic systems. A typical spatial grid size used was $h_{x}=h_{y}=0.01$ or 0.02 . An initial time step of 0.001 , which corresponds to 3.6 seconds, was used, but adaptive time

Table 3. Dimensional parameters used in the reaction-diffusion component of the model. We use the cell average packing density $2.01 \times 10^{8}$ cells $/ \mathrm{cm}^{3}$ in Casciari et al. ${ }^{15}$ to convert uptake parameters $A_{\mathrm{O}_{2}}, A_{\mathrm{gl}}, B_{\mathrm{O}_{2}}, B_{\mathrm{gl}}$ in this table to rates per unit volume.

\begin{tabular}{llll}
\hline $\mathrm{P}$ & \multicolumn{1}{c}{ Description } & \multicolumn{1}{c}{ Value } & Refs. \\
\hline Diffusion coefficients of oxygen in each region & & \\
$D_{\mathrm{O}}^{a}$ & $1 \%$ agarose & $2.15 \times 10^{-5} \mathrm{~cm}^{2} / \mathrm{s}$ & 37 \\
$D_{\mathrm{O}}^{p}$ & Proliferating region & $1.82 \times 10^{-5} \mathrm{~cm}^{2} / \mathrm{s}$ & 40 \\
$D_{\mathrm{o}}^{q}$ & Quiescent region & $1.54 \times 10^{-5} \mathrm{~cm}^{2} / \mathrm{s}$ & This work \\
$D_{\mathrm{O}}^{n}$ & Necrotic region & $1.34 \times 10^{-5} \mathrm{~cm}^{2} / \mathrm{s}$ & This work \\
Diffusion coefficients of glucose in each region & & \\
$D_{\mathrm{g}}^{a}$ & $1 \%$ agarose & $6.46 \times 10^{-6} \mathrm{~cm}^{2} / \mathrm{s}$ & 61 \\
$D_{\mathrm{g}}^{p}$ & Proliferating region & $1.1 \times 10^{-6} \mathrm{~cm}^{2} / \mathrm{s}$ & 14 \\
$D_{\mathrm{g}}^{q}$ & Quiescent region & $0.96 \times 10^{-6} \mathrm{~cm}^{2} / \mathrm{s}$ & This work \\
$D_{\mathrm{g}}^{n}$ & Necrotic region & $0.86 \times 10^{-6} \mathrm{~cm}^{2} / \mathrm{s}$ & This work \\
\hline
\end{tabular}


Table 3. (Continued)

\begin{tabular}{|c|c|c|c|}
\hline $\mathrm{P}$ & Description & Value & Refs. \\
\hline \multicolumn{4}{|c|}{ Coefficients in uptake functions } \\
\hline$c_{H}^{t}$ & $\mathrm{H}^{+}$concentration in tumor & $1 \times 10^{-4} \mathrm{mM}$ & 13 \\
\hline$A_{\mathrm{O}_{2}}$ & Oxygen uptake parameter & $1.0642 \times 10^{-16} \frac{\mathrm{mol}}{\mathrm{cell} \cdot \mathrm{s}}$ & 13,15 \\
\hline$B_{\mathrm{O}_{2}}$ & Oxygen uptake parameter & $6.0202 \times 10^{-17} \frac{\mathrm{mol} \cdot \mathrm{mM}}{\mathrm{cell} \cdot \mathrm{s}}$ & $13,15,23$ \\
\hline$A_{\mathrm{gl}}$ & Glucose uptake parameter & $1.0642 \times 10-16 \frac{\mathrm{mol}}{\mathrm{cell} \cdot \mathrm{s}}$ & $13,15,23$ \\
\hline$B_{\mathrm{gl}}$ & Glucose uptake parameter & $1.7879 \times 10^{-17} \frac{\mathrm{mol} \cdot \mathrm{m} M}{\mathrm{cell} \cdot \mathrm{s}}$ & 15,23 \\
\hline$k_{\mathrm{O}_{2}}$ & Critical oxygen concentration & $4.640 \times 10^{-3} \mathrm{mM}$ & 15 \\
\hline$k_{\mathrm{gl}}$ & Critical glucose concentration & $4.0 \times 10^{-2} \mathrm{mM}$ & 15 \\
\hline$n_{\mathrm{O}_{2}}$ & Oxygen uptake parameter & $0.55 \mathrm{mM}$ & 23 \\
\hline$n_{\mathrm{gl}}$ & Glucose uptake parameter & $0.04 \mathrm{mM}$ & 23 \\
\hline \multicolumn{4}{|c|}{ Boundary conditions } \\
\hline$c_{\mathrm{O}_{2} b c}$ & Oxygen concentration in medium & $0.2 \mathrm{mM}$ & 15,23 \\
\hline$c_{\mathrm{gl} b c}$ & Glucose concentration in medium & $25 \mathrm{mM}$ & 29 \\
\hline \multicolumn{4}{|c|}{ Quiescent region thresholds } \\
\hline$t h_{q \mathrm{O}_{2}}$ & Critical oxygen concentration & $0.0625 \mathrm{mM}$ & This work \\
\hline$t h_{q \mathrm{gl}}$ & Critical glucose concentration & $1.17 \mathrm{mM}$ & This work \\
\hline \multicolumn{4}{|c|}{ Necrosis thresholds } \\
\hline$t h_{n \mathrm{O}_{2}}$ & Critical oxygen concentration & $0.0500 \mathrm{mM}$ & 15 , this work \\
\hline$t h_{n \mathrm{gl}}$ & Critical glucose concentration & $0.0055 \mathrm{mM}$ & 15 \\
\hline
\end{tabular}

stepping based on the number of iterations could increase or decrease this. After convergence of the nutrient solve step, we check the level of nutrients in order to determine whether cells near the boundary in the proliferating region have to be transformed to the quiescent region and the $\mathcal{P}-\mathcal{Q}$ interface has to be updated. For this purpose the nutrient level at the center of the cell is used, and interpolation to and from grid to cell is done as described in DO. Knowing the level of nutrients at the cell sites we have three choices for determining the $\mathcal{P}-\mathcal{Q}$ interface: either oxygen or glucose must be below a threshold, or both must be below their thresholds. Here we use the oxygen level as the critical nutrient that determines the interface. An outline of the complete computational algorithm is as follows.

\subsection{The computational algorithm}

Step 0. Initialization.

Step 0.1 Set rectangular grid for ADI algorithm that determines nutrient concentrations, and initialize nutrient values.

Step 0.2 Initialize cell-based component by randomly placing cells in the proliferating domain.

Step 0.3 Use these cells to create boundaries of continuum regions. Create mesh in continuum region.

Step 0.4 Let the system relax to mechanical equilibrium, and allow the nutrient profiles to reach equilibrium given the initial configuration of the tumor. 
Step 1. Locate all cells and continuum mesh that are within a given distance from cell $i$.

Step 2. Deformation and translation of cells.

Step 2.1 Find all the forces that act on the cell, $\mathbf{F}^{\text {net }}$ from each of the neighbor cells found in [Step 1].

Step 2.2 Deform the three axes of the ellipsoid according to (3.2)-(3.5) and, based on the nutrient levels and stress magnitude, allow cells to grow.

Step 2.3 Move the cells according to the force balance equation (3.7).

Step 3. Communicate between continuum and cell-based regions by transferring data at region boundaries.

Step 3.1 Determine location, magnitude, and direction of force exerted by cells onto continuum boundary.

Step 3.2 Using the finite element interpolation functions that are valid on the boundary elements, distribute point forces from cells onto nodes of elements. This nodal force distribution is used as a natural boundary condition for the stress distribution in the continuum regions.

Step 4. Calculate the stress in continuum regions. Remesh continuum regions if current mesh becomes severely distorted.

Step 5. Divide cells when their volume reaches $2 V_{0}$. Axis of division is determined by all forces acting on the cell. Once cell division occurs, we allow the cells to reach a new mechanical equilibrium that accounts for the newly added cells.

Step 6. Update the nutrient levels using the ADI method. This does not need to be done every iteration.

Step 7. Update continuum regions.

Step 7.1 Check the nutrient level for cells near the $\mathcal{P}-\mathcal{Q}$ interface to determine if the nutrient levels are below the threshold defining quiescent region, and if so, find the cells in this region. These cells will be removed from the cell-based model and will become represented by the continuum model.

Step 7.2 Update the new interface between the proliferating region and quiescent region. i.e. generate a new mesh on the interior $(\mathcal{Q}+$ $\mathcal{N})$ region and interpolate the data from the old mesh to this new mesh or from the cell-based model to the new mesh, as appropriate. (See Fig. 3.) A least squares approximation is used for data interpolation.

Step 7.3 Nutrient threshold values are used to determine new $\mathcal{Q}-\mathcal{N}$ boundary.

Step 8. Go to [Step 1]. 


\section{Computational Results}

We first investigate the growth of a tumor in the absence of the outer agarose gel, which is similar to experiments in which a tumor is grown in suspension. We use an initial configuration of the tumor in which the proportions of the three regions are specified, but which are not solutions of the full system, and allow the system to evolve. The initial regions are circularly symmetric, and both the quiescent region and the necrotic core are treated as continua. In Fig. 6(a) we show the initial configuration and three later times, and in Fig. 6(b) we show the corresponding oxygen and glucose concentrations. In (a) proliferating cells transform to quiescent cells and quiescent cells to necrotic cells when the oxygen level interpolated to the cell centroid drops below specified thresholds. It is clear in (a) that the tumor grows asymmetrically, and this is reflected in the asymmetric nutrient profiles in (b). This asymmetry arises in part from the dynamics and in part from the numerical procedures. The former arises from small variations in size and hence in division time, which in turn biases nutrient profiles and in turn reinforces the differences
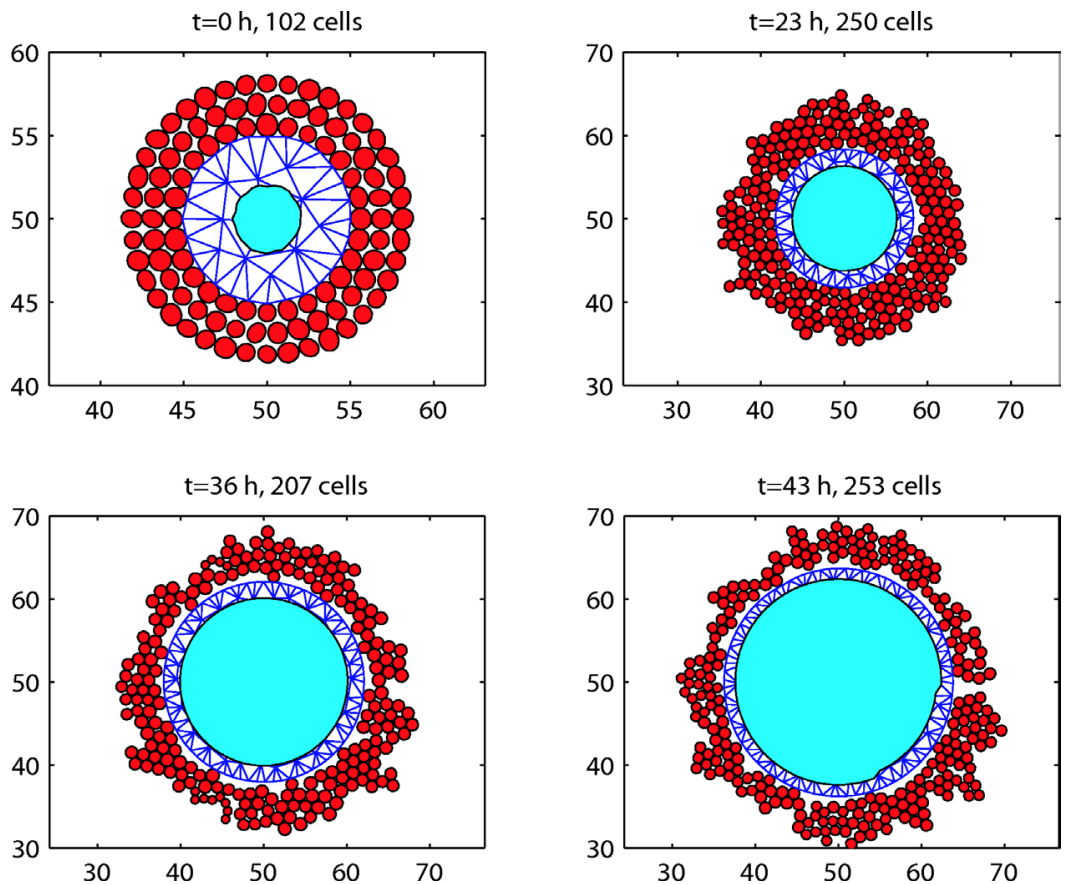

(a)

Fig. 6. (a) Evolution of a tumor spheroid in the absence of the outer gel. $\mathcal{N}$ is represented by the inner light blue region; it is enclosed by the continuum quiescent region $(\mathcal{Q})$ and the outer cell-based region. Here and in the remaining figures the space unit is 10 microns. (b) The time evolution of the oxygen and glucose levels. Upper panels: $t=1 \mathrm{hr}$, lower panels: $t=43 \mathrm{hr}$. The parameters used are: $c^{+}=1.16089 \times 10^{-8} \mathrm{~mm} /(\mathrm{min} \cdot \mathrm{nN}), \sigma^{-}=-400 \mathrm{nN}, A_{\mathrm{O}_{2}}=3.08014, B_{\mathrm{O}_{2}}=$ $6.97 \times 10^{-2}, A_{\mathrm{gl}}=8.00038, B_{\mathrm{gl}}=2.07 \times 10^{-2}$ (dimensionless values). 

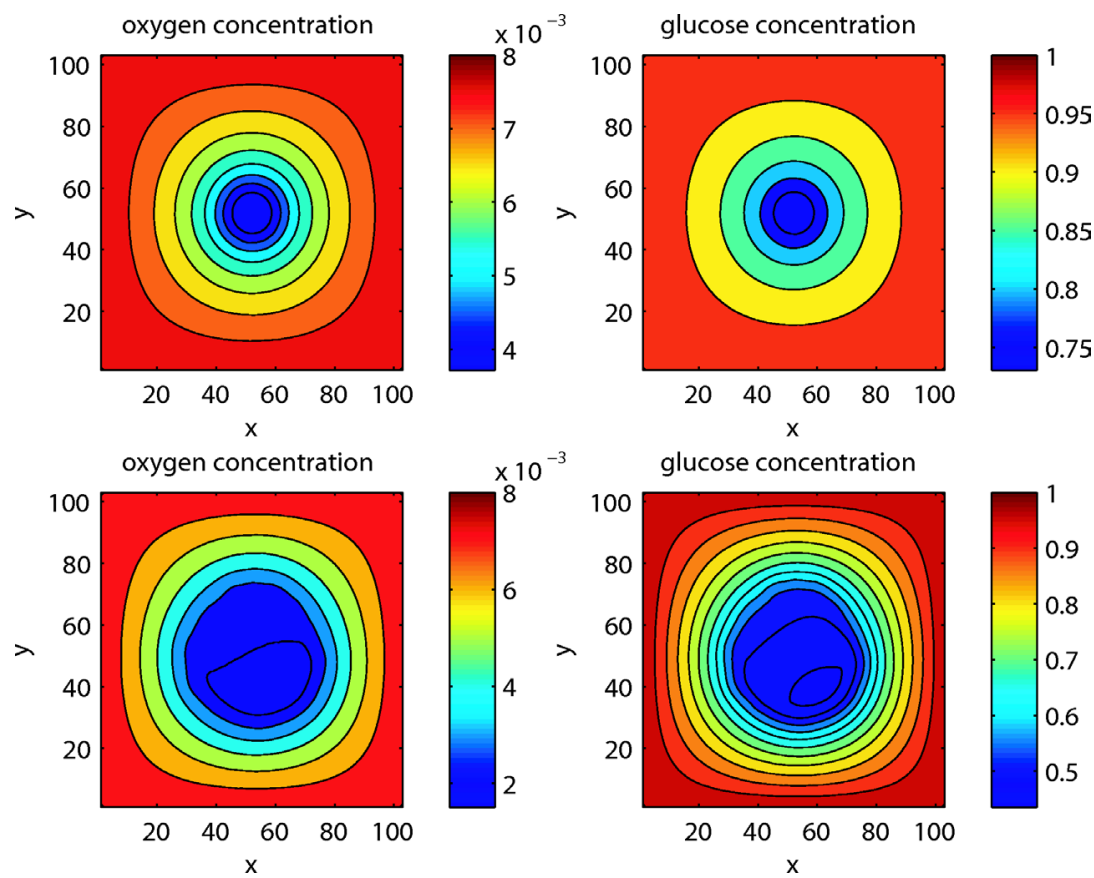

(b)

Fig. 6. (Continued)

in growth. Since there is no restraining force outside the proliferating region there is no mechanism for reducing this source of asymmetry. The latter arises because the cell states are updated sequentially, which introduces an asymmetry into the growth. Further tests to establish the contribution of each source are needed, but results shown later for growth in the presence of an outer gel suggest that the former is the primary source of the asymmetry.

Figure 7 shows the growth in time of the diameters of the proliferating, quiescent and necrotic regions of the tumor. Here and hereafter the diameters of the $\mathcal{Q}$ and $\mathcal{N}$ regions were determined by taking the average distance from the spheroid center to each node on each contact boundary, i.e. the $\mathcal{P}-\mathcal{Q}$ and the $\mathcal{Q}-\mathcal{N}$ interface, respectively. Similarly the diameter of a tumor is defined as $\max _{i} 2.0\left(d_{c}^{i}+r_{c}^{i}\right)$ where $d_{c}^{i}$ is the distance from the center of the $i$ th cell in $\mathcal{P}$ region to the spheroid center and $r_{c}^{i}=0.5 *\left(r a_{c}^{i}+r b_{c}^{i}\right)$, where $r a_{c}^{i}, r b_{c}^{i}$ are the half length of axial lengths in two major axis $\mathbf{a}, \mathbf{b}$. One sees in Fig. 7 that the initial configuration is not a solution of the governing equations, since there is an initial rapid increase in the diameter of the quiescent and necrotic regions. Some of the irregularity in the expansion of the quiescent region is due to the fact that the $\mathcal{Q}-\mathcal{P}$ interface is advanced one layer of cells at a time. That is, rather than advancing the interface on a cell-by-cell basis as cells cross threshold, we wait until all cells within a nominal diameter of the 


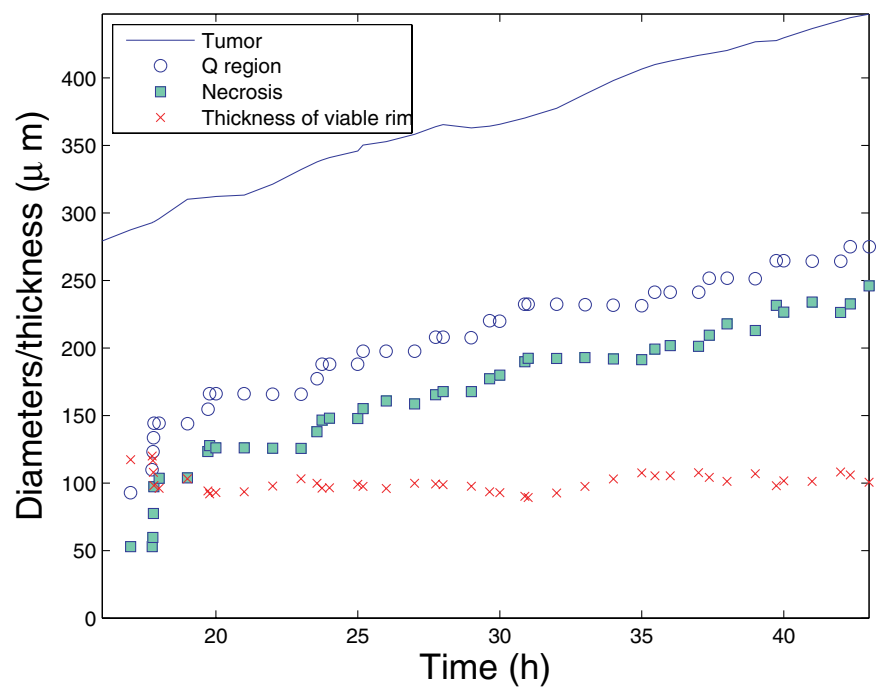

Fig. 7. The evolution of the diameter of the tumor, the quiescent region, and the necrotic region, as well as the thickness of the viable region in the tumor in Fig. 6 as a function of time.

existing interface cross threshold, and then advance the entire layer. This is done for computational expediency at present, but refinement of this step is possible. Similarly, the irregularity of the $\mathcal{Q}-\mathcal{N}$ interface is due to the fact that we do not re-mesh every time step, and therefore the motion of the interface lags the change in the nutrient threshold. Again, this can be refined.

One also sees in Fig. 7 that after about 20 hours the system settles into a quasistatic growth regime in which the diameter of the tumor continues to increase at a constant rate. It is noteworthy that after this initial transient the thickness of the viable region, which is defined as the tumor radius minus the radius of the necrotic region, remains essentially constant at about 100 microns. This linear growth regime, in which the thickness of the viable region is essentially constant, is also seen experimentally in many, but not all, tumor spheroids (cf. Fig. 2 in Ref. 40). As we will see in the following, the thickness of the viable region remains more or less constant, even when the tumor grows more slowly due to elastic forces on the tumor from the surrounding medium.

To understand the effect of stress on the growth rate of a tumor, we studied the growth of spheroids in an agarose gel of four different stiffness, as measured by the Young's moduli, using other parameter values as given in the tables, and initial conditions as in the free suspension. Again this initial configuration is not a solution of the governing equations, and is first allowed to relax both mechanically and with respect to the nutrient distribution. One sees in Fig. 8 that the shape of a tumor spheroid embedded in an agarose gel is relatively symmetric and much more regular than the shape of a tumor growing in free suspension (cf. Fig. 6). 

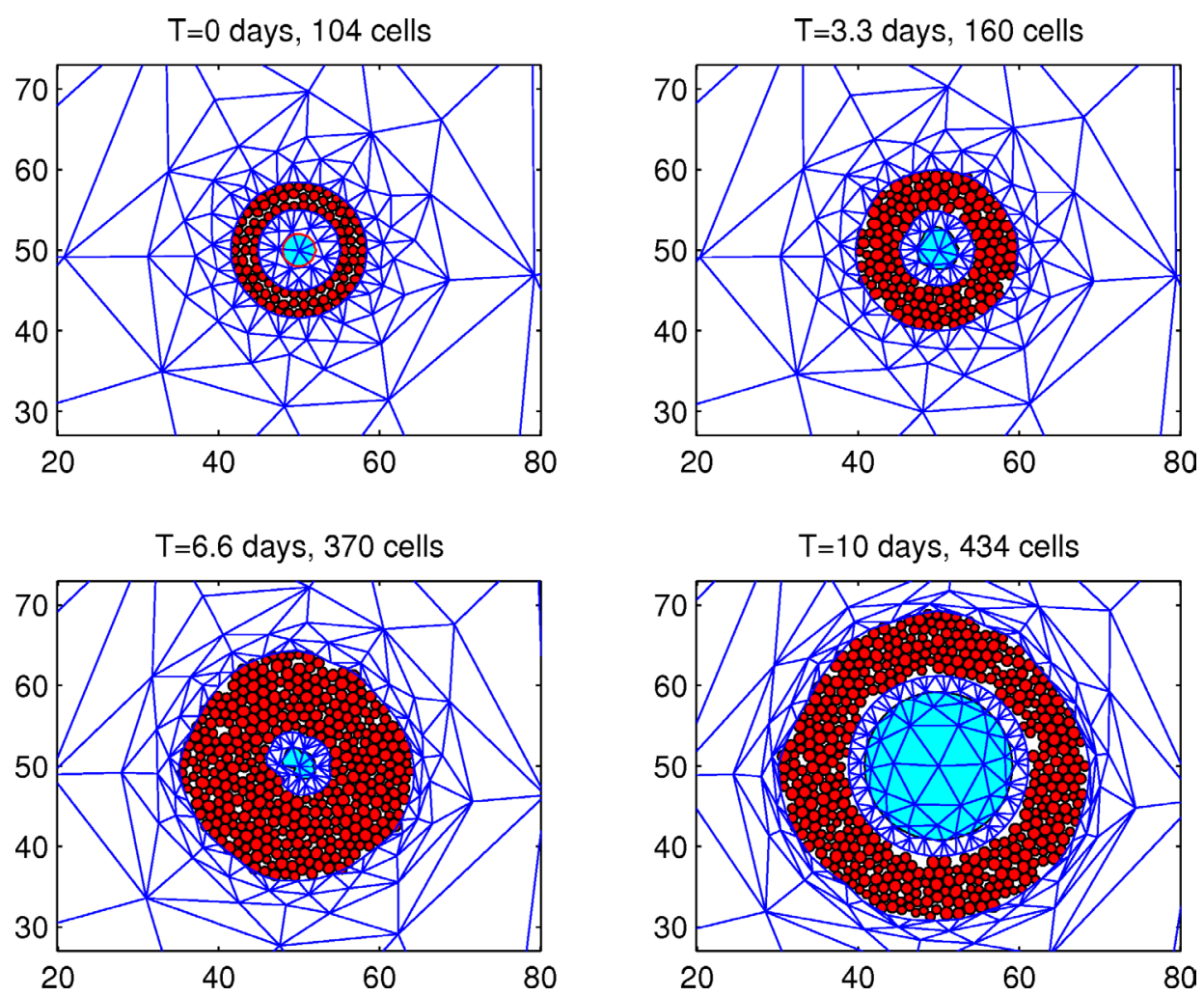

Fig. 8. The growth of a tumor embedded in an agarose gel of Young's modulus $E^{a}=200 \mathrm{mPa}$. Note that the thickness of the viable rim has stabilized by about 7 days.

The necrotic region, proliferating region, and overall tumor growth profiles are governed by nutrient uptake, which is primarily dependent on the proliferating region. As the tumor grows by expanding the proliferating region, the level of nutrients at the center decreases. When the oxygen in the interior-most layer of cells drops below the threshold that determines the transition to quiescence, a layer of cells is converted to the $\mathcal{Q}$ continuum. As the tumor grows further the level drops below the $\mathcal{Q} \rightarrow \mathcal{N}$ threshold and quiescent cells become necrotic. However, as shown in Fig. 9, after the initial transient the thickness of the viable region stabilizes at about 100 microns, independent of the stiffness of the gel. However, the stiffness does have a significant effect on the overall growth rate of the tumor. In Figs. 9(a)-9(d) the Young's moduli are 10, 20, 80 and $200 \mathrm{MPa}$, respectively, and the corresponding growth rates are 44, 44, 33, and 28 microns/day, respectively. Thus a relatively compliant gel shows little difference in growth from growth in suspension, but when the stiffness is large enough that the stress exerted by the gel exceeds $\alpha$ in Fig. 4, growth slows. Since the computational domain $\Omega$ in which the tumor and gel are contained is bounded, and since the boundary of $\Omega$ is fixed, the 


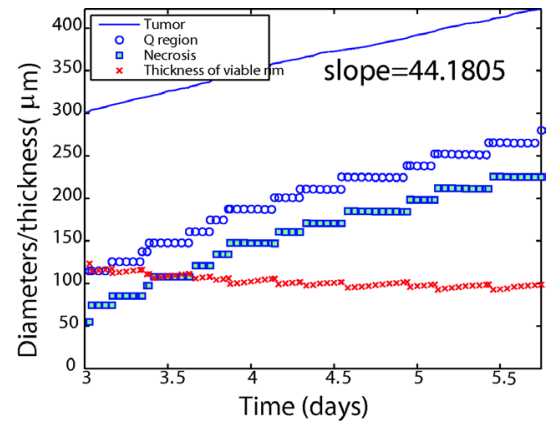

(a)

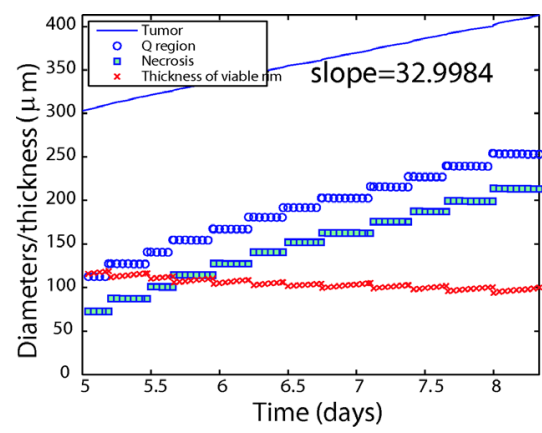

(c)

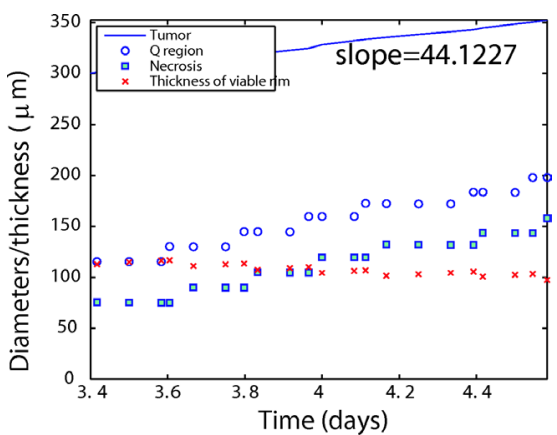

(b)

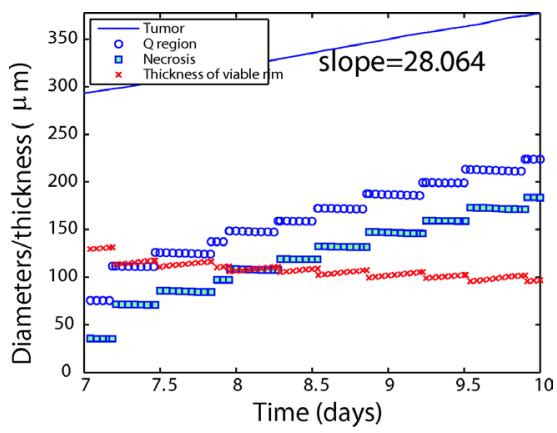

(d)

Fig. 9. The growth rates of tumors in gels of increasing stiffness. Panels (a)-(d) correspond to Young's moduli $E^{a}$ of the gel of 10, 20, 80, and $200 \mathrm{MPa}$, respectively, while other parameters are as in the tables. Despite a twenty-fold difference in stiffness, the thickness $(x)$ of the viable rim is essentially constant, even though the diameters of the tumor (solid line), the quiescent region, and necrotic region increase. The steps in the diameters of $\mathcal{Q}$ and $\mathcal{N}$ region occur for the reasons given in the text.

stress effect on growth would also be seen at sufficiently large times for the more compliant gels.

\section{Discussion}

In the hybrid model described herein we treat the quiescent and necrotic tumor regions as continua and the region in which tumor cells actively proliferate as ellipsoidal single cells. As a result this model can be used to address numerous experiments which one may not be able to address by discrete-cell off-lattice or continuum models alone. We can thereby increase our understanding of tumor growth and the effect of the tumors micro-environment on that growth. We have only discussed a $2 \mathrm{D}$ version of the model here, but it is important to consider a model of the full $3 \mathrm{D}$ tumor environment as there can be large differences in intracellular signaling, intercellular interactions, and drug responses between 2D tumor cell cultures and 
3D cultures. ${ }^{51}$ We are currently developing a 3D version of the techniques described herein, and the results from the 3D model will be reported elsewhere.

A major advantage of the approach described here is that details of intracellular dynamics and cell-cell interactions in the proliferating region are easily included without compromising the numerical tractability of the method. For example, it is easy to introduce changes in cell-level parameters such as adhesion, growth rates, etc and investigate their consequences. One can also include chemotaxis in the model, which in turn will allow us to consider tumor cell shedding, a phenomenon of particular importance in the dynamics of highly invasive glioma tumors. ${ }^{27}$ In addition, it has been shown that upon disrupting cell-cell adhesion and protease function in tumor cell lines, the cells can switch modes from collective motility to single-cell amoeboid motility. ${ }^{58}$ This plasticity in motility mechanisms depends on the tumor environment, and the present model is ideally suited to investigate these highly-localized effects.

Further work on the effect of stress on tumor growth will involve a comparison of our numerical results with the experiments of Helmlinger et $a .^{29}$ in detail. Doing so will allow us to more accurately determine how material properties of the necrotic region and forms of the nutrient uptake functions affect tumor growth. In addition, in the numerical results described herein, we assume that the effect of stress on growth is linear and isotropic and that compressive and tensile stresses have the same effect. It is unlikely that this is the case, and a detailed comparison of numerical results to experimental findings will allow us to more accurately determine the functional form for the dependence of growth of spheroids and in vivo tumors on stress.

In light of the flexibility and applicability of the hybrid model, further mathematical analysis and numerical validation of the full hybrid model is warranted, and the results of this analysis will be reported in a future paper.

\section{Acknowledgments}

Research supported by NSF grants 0317372 and 0517884, and by the Minnesota Supercomputing Institute.

\section{References}

1. D. Ambrosi and F. Mollica, On the mechanics of a growing tumor, Int. J. Engng. Sci. 40 (2002) 1297-1316.

2. - The role of stress in the growth of a multicell spheroid, J. Math. Biol. 48 (2004) 477-499.

3. R. Araujo and D. McElwain, A history of the study of solid tumour growth: The contribution of mathematical modelling, Bull. Math. Biol. 66 (2004) 1039-1091.

4. - A linear-elastic model of anisotropic tumour growth, Euro. J. Appl. Math. 15 (2004) 365-384.

5. R. P. Araujo and D. L. S. McElwain, New insights into vascular collapse and growth dynamics of solid tumors, J. Theor. Biol. 228 (2004) 335-346. 
6. R. P. Araujo and D. L. S. McElwain, The role of mechanical host-tumor interactions in the collapse of tumour blood vessels and tumour growth dynamics, J. Theor. Biol. 238 (2006) 817-827.

7. C. Athale, Y. Mansury and T. S. Deisboeck, Simulating the impact of a molecular "decision-process" on cellular phenotype and multicellular patterns in brain tumors, J. Theor. Biol. 233 (2005) 469-481.

8. R. J. Atkin and R. E. Craine, Continuum theories of mixtures: Basic theory and historical development, Quart. J. Mech. Appl. Math. 29 (1976) 209-244.

9. T. Berdyyeva, C. Woodworth and I. Sokolov, Human epithelial cells increase their rigidity with ageing in vitro: Direct measurements, Phys. Med. Biol. 50 (2005) 81-92.

10. R. M. Bowen, Incompressible porous-media models by use of the theory of mixtures, Int. J. Engng. Sci. 18 (1980) 1129-1148.

11. C. J. W. Breward, H. M. Byrne and C. E. Lewis, A multiphase model describing vascular tumor growth, Bull. Math. Biol. 65 (2003) 609-640.

12. H. M. Byrne, J. R. King, D. L. S. McElwain and L. Preziosi, A two-phase model of solid tumour growth, Appl. Math. Lett. 16 (2003) 567-573.

13. J. Casciari, S. Sotirchos and R. Sutherland, Variations in tumor cell growth rates and metabolism with oxygen concentration, glucose concentration, and extracellular $\mathrm{pH}$. J. Cell. Physiol. 151 (1992) 386-394.

14. J. J. Casciari, S. V. Sotirchos and R. M. Sutherland, Glucose diffusivity in multicellular tumor spheroids, Cancer Res. 48 (1988) 3905-3909.

15. - Mathematical modelling of microenvironment and growth in EMT6/Ro multicellular tumour spheroids, Cell Prolif. 25 (1992) 1-22.

16. C. Y. Chen, H. M. Byrne and J. R. King, The influence of growth-induced stress from the surrounding medium on the development of multicell spheroids, J. Math. Biol. 43 (2001) 191-220.

17. A. Cho, Life's patterns: No need to spell it out?, Science 303 (2004) 782-783.

18. J. C. Dallon and H. G. Othmer, How cellular movement determines the collective force generated by the Dictyostelium discoideum slug, J. Theor. Biol. 231 (2004) 203-222.

19. D. Drasdo, Buckling instabilities of one-layered growing tissues, Phys. Rev. Lett. 84 (2000) 4244-4247.

20. D. Drasdo, S. Dormann, S. Hoehme and A. Deutsch, Cell-Based Models of Avascular Tumor Growth (Birkhauser, 2003).

21. D. Drasdo and G. Forgacs, Modeling the interplay of generic and genetic mechanisms in cleavage, blastulation, and gastrulation, Develop. Dyn. 219 (2000) 182-191.

22. D. Drasdo and S. Hohme, A single-cell-based model of tumor growth in vitro: Monolayers and spheroids, Phys. Biol. 2 (2005) 133-147.

23. J. Freyer and R. Sutherland, A reduction in the in situ rates of oxygen and glucose consumption of cells in emt6/ro spheroids during growth, J. Cell Physiol. 124 (1985) $516-524$.

24. - Regulation of growth saturation and development of necrosis in emt6/ro multicellular spheroids by the glucose and oxygen supply, Cancer Res. 46 (1986) 3504-3512.

25. J. Galle, G. Aust, G. Schaller, T. Beyer and D. Drasdo, Individual cell-based models of the spatial-temporal organization of multicellular systems - achievements and limitations, Cytometry A 69A (2006) 704-710.

26. J. Galle, M. Loeffler and D. Drasdo, Modeling the effect of deregulated proliferatin and apoptosis on the growth dynamics of epithelial cell populations in vitro, Biophys. J. 88 (2005) 62-75. 
27. A. Giese, R. Bjerkvig, M. Berens and M. Westphal, Cost of migration: Invasion of malignant gliomas and implications for treatment, J. Clinical Oncol. 21 (2003) 16241636.

28. V. D. Gordon, M. T. Valentine, M. L. Gardel, D. Andor-Ardo, S. Dennison, A. A. Bogdanov, D. A. Weitz and T. S. Deisboeck, Measuring the mechanical stress induced by an expanding multicellular tumor system: A case study, Exp. Cell Res. 289 (2003) $58-66$.

29. G. Helmlinger, P. A. Netti, H. C. Lichtenbeld, R. J. Melder and R. K. Jain, Solid stress inhibits the growth of multicellular tumor spheroids, Nature Biotech. 15 (1997) $778-783$.

30. A. Hoger, Residual stress in an elastic body: A theory for small strains and arbitrary rotations, J. Elasticity 31 (1993) 1-24.

31. A. F. Jones, H. M. Byrne, J. S. Gibson and J. W. Dold, A mathematical model of the stress induced during avascular tumour growth, J. Math. Biol. 40 (2000) 473-499.

32. K. Landman and C. Please, Tumour dynamics and necrosis: Surface tension and stability, Math. Med. Biol. 18 (2001) 131-158.

33. J. Landry, J. Freyer and R. Sutherland, Shedding of mitotic cells from the surface of multicell spheroids during growth, J Cell Physiol. 106 (1981) 23-32.

34. Y. Mansury, M. Diggory and T. S. Deisboeck, Evolutionary game theory in an agentbased brain tumor model: Exploring the "genotype-phenotype" link, J. Theor. Biol. 238 (2006) 146-156.

35. Y. Mansury, M. Kimura, J. Lobo and T. S. Deisboeck, Emerging patterns in tumor systems: Simulating the dynamics of multicellular clusters with and agent-based spatial agglomeration model, J. Theor. Biol. 219 (2002) 343-370.

36. N. V. Mantzaris, S. Webb and H. G. Othmer, Mathematical modeling of tumorinduced angiogenesis, J. Math. Biol. 49 (2004) 111-187.

37. M. McCabe and T. Laurent, Diffusion of oxygen, nitrogen and water in hyaluronate solutions, Biochim. Biophys. Acta 399 (1975) 131-138.

38. J. Moreira and A. Deutsch, Cellular automaton models of tumor development: A critical review, Adv. Complex Syst. 5 (2002) 247-267.

39. W. Mueller-Klieser, Tumor biology and experimental therapeutics, Crit. Rev. Oncol. Hematol. 36 (2000) 123-139.

40. W. F. Mueller-Klieser and R. M. Sutherland, Oxygen consumption and oxygen diffusion properties of multicellular spheroids from two different cell lines, Adv. Exp. Med. Biol. 180 (1984) 311-321.

41. H. G. Othmer, On the significance of finite propagation speeds in multicomponent reacting systems, J. Chem. Phys. 64 (1976) 460-470.

42. E. Palsson and H. G. Othmer, A model for individual and collective cell movement in Dictyostelium discoideum, Proc. Nat. Acad. Sci. 97 (2000) 10448-10453.

43. C. Please, G. Pettet and D. McElwain, A new approach to modelling the formation of necrotic regions in tumors, Appl. Math. Lett. 11 (1997) 89-94.

44. C. Please, G. Pettet, D. McElwain, N. Bellomo and M. Chaplain, Avascular tumour dynamics and necrosis, Math. Mod. Meth. Appl. Sci. 9 (1999) 569.

45. M. Radmacher, Measuring the elastic properties of biological samples with the AFM, IEEE Eng. Med. Biol. Mag. 16 (1997) 47-57.

46. K. A. Rejniak, A single-cell approach in modeling the dynamics of tumor microregions, Math. Biosci. Eng. 2 (2005) 643-655.

47. T. Roose, P. Netti, L. Munn, Y. Boucher and R. Jain, Solid stress generated by spheroid growth estimated using a linear poroelastisity model, Microvascular Res. 66 (2003) 204-212. 
48. C. Rotsch, F. Braet, E. Wisse and M. Radmacher, AFM imaging and elasticity measurements on living rat liver macrophages, Cell Biol. Int. 21 (1997) 685-696.

49. G. Schaller and M. Meyer-Hermann, Multicellular tumor spheroid in an off-lattice Voronoi-Delaunay cell model, Phys. Rev. E 71 (2005) 051910.

50. B. I. Shraiman, Mechanical feedback as a possible regulator of tissue growth, Proc. Natl. Acad. Sci. USA 102 (2005) 3318-3323.

51. K. S. M. Smalley, M. Lioni and M. Herlyn, Life isn't flat: Taking cancer biology to the next dimension, In Vitro Cell Dev. Biol. Anim. 42 (2006) 242-247.

52. A. M. Stein, T. Demuth, D. Mobley, M. Berens and L. M. Sander, A mathematical model of glioblastoma tumor spheroid invasion in an three-dimensional in vitro experiment, Biophys. J. 92 (2007) 356-365.

53. R. Sutherland, B. Sordat, J. Bamat, H. Gabbert, B. Bourrat and W. Mueller-Klieser, Oxygenation and differentiation in multicellular spheroids of human colon carcinoma, Cancer Res. 46 (1986) 5320-5329.

54. S. Turner and J. A. Sherratt, Intercellular adhesion and cancer invasion: A discrete simulation using the extended Potts model, J. Theor. Biol. 216 (2002) 85-100.

55. J. Ward and J. King, Mathematical modelling of avascular-tumour growth. II: Modelling growth saturation, IMA J. Math. Appl. Med. Biol. 16 (1999) 171-211.

56. - Mathematical modelling of drug transport in tumour multicell spheroids and monolayer cultures, Math. Biosci. 181 (2003) 177-207.

57. J. P. Ward and J. R. King, Mathematical modelling of avascular tumour growth., IMA J. Math. Appl. Med. Biol. 14 (1997) 39-69.

58. K. Wolf and P. Friedl, Molecular mechanisms of cancer cell invasion and plasticity, Br. J. Dermatol. 154 (2006) 11-15.

59. H. Wu, T. Kuhn and V. Moy, Mechanical properties of 1929 cells measured by atomic force microscopy: Effects of anticytoskeletal drugs and membrane crosslinking, Scanning 20 (1998) 389-397.

60. L. Zhang, C. A. Athale and T. S. Beisboeck, Development of a three-dimensional multiscale agent-based tumor model: Simulating gene-protein interaction profiles, cell phenotypes and multicellular patterns in brain cancer, J. Theor. Biol. 244 (2007) 96-107.

61. T. Zhang and H. H. P. Fang, Effective diffusion coefficients of glucose in artificial biofilms, Environmental Tech. 26 (2005) 155-160(6). 